\title{
URBAN TRAFFIC CONTROL: A STREAMING MULTIMEDIA APPROACH
}

\author{
C. E. Palau, M. Esteve, J.Martínez, B. Molina, I. Pérez \\ Communications Department. ETSIT, Universidad Politécnica de Valencia \\ Camino de Vera S/N Valencia 46022 (SPAIN) \\ \{cpalau, mesteve\}@dcom.upv.es; \{jamarno,benmomo,ispello0\}@doctor.upv.es
}

\begin{abstract}
Urban traffic control systems have based their technological infrastructure both on advanced analogical close-circuit television systems (TVCC) and point-topoint links, providing low-scalable and very expensive systems. The main goal of an urban traffic monitoring system is to capture, send, play and distribute video information from the streets of a certain city. Current digitalization process of video networks, and the research carried out in the field of streaming media, has led vendors to present proprietary hardware and software solutions resulting in a strong dependency among their customers. The existence of open standards for video encoding and protocols for streaming media transmission over IP networks has led us to propose this system. The work presents an open urban traffic control system which bases its design on COTS philosophy for hardware and software, as well as open source and standardized protocols. The proposed system is a suitable solution in terms of scalability, cost, interoperability and performance for traffic control systems. Futhermore, its architecture can be easily adapted to other video applications and tools.
\end{abstract}

\section{INTRODUCTION}

Vision-based systems for traffic management have been installed in many cities all around the world. [1][2] The information collected by these systems is sent to the central Traffic Management Centre (TMC), where it is treated in different ways: played on screen monitors, saved in video tapes and sometimes processed and automatically analysed for different uses, such as fast incident detection without human monitoring, estimation of travel times between various points, lane usage, heavy vehicle count or detailed traffic condition information for public use. The digital treatment of these video signals is required for the processing of the information and for using artificial vision techniques.

Traffic management and surveillance systems based in the utilisation of cameras need high quality in the video signals. Usually, CCTV techniques have been used for the implantation of such systems, due to the provision of high quality video signals [3]. The main drawbacks of CCTV systems are lack of scalability, cost of cabling network, and the difficulty in providing video information to the public through Internet. Other network based solutions have been adopted for traffic video surveillance systems: (i) using web cameras reducing the quality of the video signal and a general purpose TCP/IP network or (ii) using a digital cabling network to reduce cabling costs and improve scalability problems [4]. But currently, no system targets all drawbacks by using a high-speed TCP/IP network with QoS, keeping the quality of the video signal using MPEG4 compression standard, video streaming techniques and providing an improvement in the scalability by sharing links by multiple streams [5][6].

Streaming media systems transmit media such as video, audio or any additional data over wired and wireless networks, but a key difference among these kinds of systems is whether the media is distributed in real-time or on-demand. Multimedia is already being distributed in the Internet and in many other communication networks and systems [3]. Using many different products like RealNetworks RealSystem, Nullsoft's SHOUTcast, Windows Media or Apple's Quicktime the idea of broadcasting has been brought to the web. Besides there are other multimedia applications based on the requests of the customers which are generally known as Video on-Demand systems (VoD). A VoD system allows customers to select movies from a large menu in order to view them (e.g., at home) at times of their choosing. This has motivated advancements in a number of research areas including techniques for video compression, error-resilient video delivery for lossy packet networks and media networking system design coupling media delivery and networking [3-6]. This paper is related with the latest research area, we present a traffic management system for a large city in Spain based in media streaming techniques.

In this paper we describe the system that has been developed during the last years, and which main features are: 


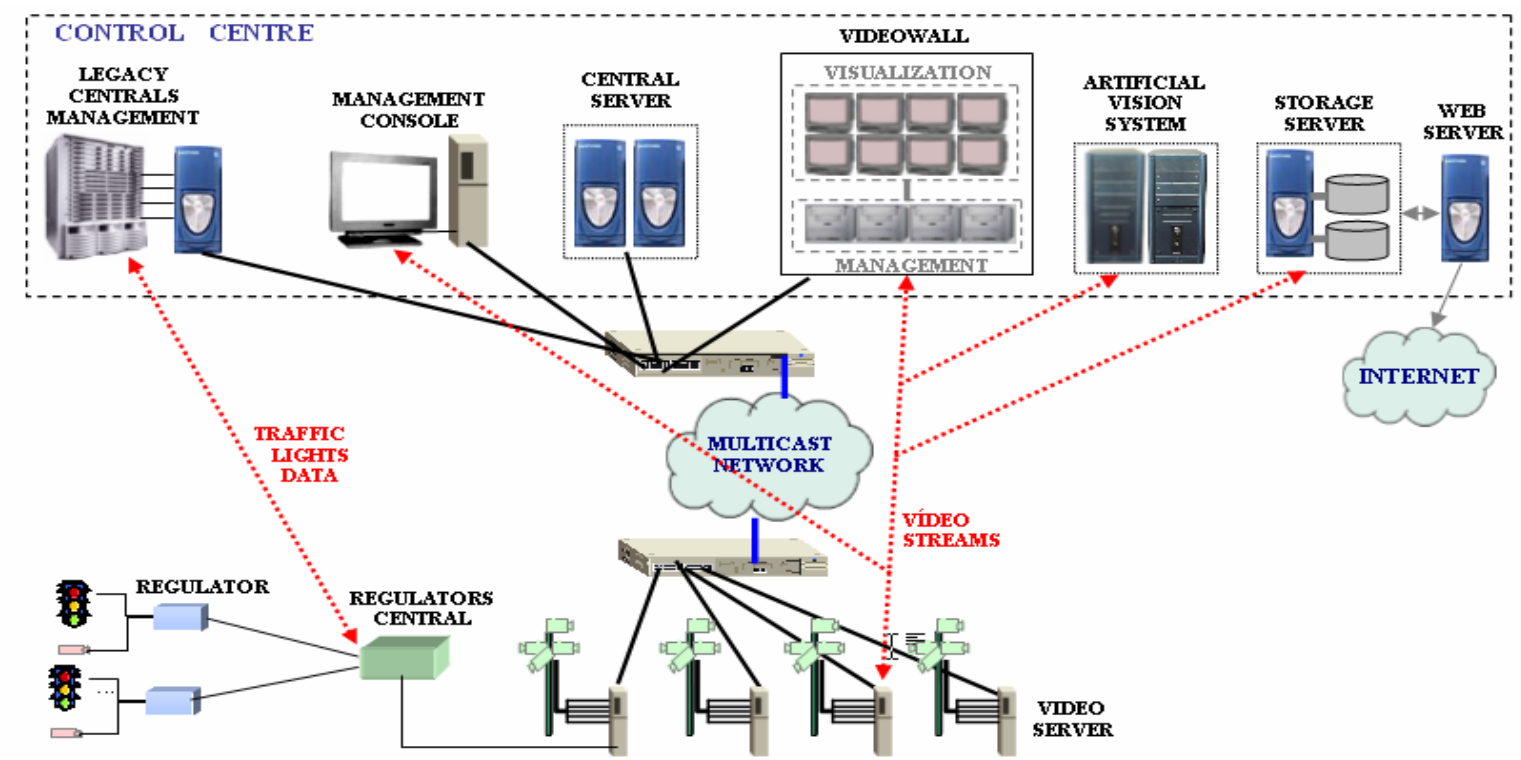

Figure 1. Architecture of the system

1) TCP/IP network with redundancy in the topology managed by the Spanning Tree protocol.

2) Capacity to hold over a thousand high quality video streams from the video cameras.

3) Utilization of MPEG4 compression standard to send the video streams.

4) Utilization of real-time protocols (RTP).

5) Multicast delivery in order to play the same flow in more than one terminal.

The reminder of this paper is structured as follows. Section 2 presents an outline of the system, the architecture and main components. Section 3 analyses in detail the planned infrastructure to support the video traffic information. In section 4 we present the capture process of urban traffic video information. Section 5 shows the video streams management and reproduction application that has been designed. Finally, in section 6 we outline the conclusions and future work related with the advanced traffic management system of the city of Valencia.

\section{OUTLINE OF THE SYSTEM}

The urban traffic control system has been developed using COTS philosophy, open source software over Linux and allowing the distribution of multimedia data via RTP (Real Time Transport Protocol).

Video streaming is the key element of the system. Among the different open alternatives to develop our system, we have chosen to develop a customized Videolan Client [7]. Videolan is a complete software solution for video streaming, inititally developed by the 'Ecole Centrale Paris' and afterwards by different developers all over the world under the GNU public license. VLC supports a great number of video and audio codecs (MPEG-1/2/4, DivX, Xvid, WMV, H.264, etc), as well as common streaming protocols (UDP, RTP, RTSP, HTTP and MMSH). Its modular and open source implementation approach makes it suitable for the deployment of custom video streaming projects whereas close proprietary solutions present management difficulties, both at technological and legal scopes.

The manufacturers of video server devices have adapted their hardware video servers to the new digital transmission networks and the latest video compression standards. Digital technologies and video standards evolve quickly and devices become rapidly obsolete. The result is that hardware-based video servers are very expensive and become obsolete before their amortization.

Current COTS hardware with specialized software is able to implement the functions of a video server. The resulting system does scale economically and results in a cost effective video server solution that is able to upgrade its functions as new technologies and standards appear. One drawback of this combination is that specialized software can result expensive in a mid-size project.

Figure 1 shows the architecture of the system, in which the video data of the urban traffic, together with data from different sensors, are captured and sent to the Traffic Management Center (TMC), where they are processed, played and stored. The video streaming visualization can be done locally (with the maximum quality) or through Internet providing access to the $\mathrm{VoD}$ Server that has been developed within the system and using RTP as communication protocol. Only a certain group of cameras can be accessed through the web server via Internet. Video sent through the web server is transcoded in order to reduce bandwidth consumption. 


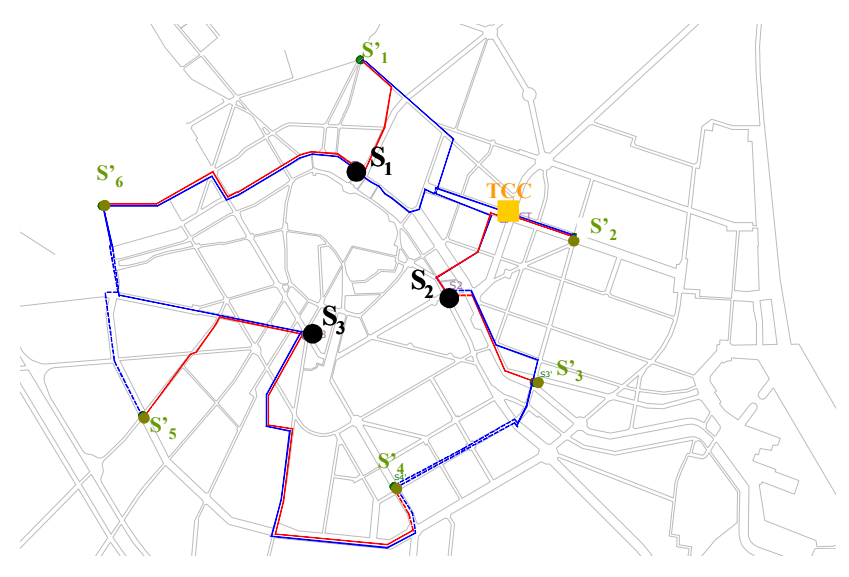

Figure 2. Scheme of the core and distribution network

\section{NETWORK INFRASTRUCTURE}

The communication network is implemented in a threelayer model approach:

- Core Network: it is a high-speed switched IP network on top of a QoS multicast aware Gigabit Ethernet LAN, working over optical fiber links. It consists of three switches deployed in the city (S1, S2 and S3) and another switch located in the TMC.

- Distribution Network: it is deployed using Fast Ethernet Switches, with Gigabit ports connected to the core network. There are currently six $\left(\mathrm{S}_{1}{ }^{\prime}\right.$ to $\left.\mathrm{S}_{6}{ }^{\prime}\right)$ distribution switches.

- Access Network: it can be subdivided into two sublayers: (i) video access network, composed of the remote cameras, the remote video servers and the display and storage subsystem placed (TMC) and (ii) data access network, formed by remote sensors mainly related to traffic-lights- , sensor-multiplexing centrals and the central management system (TMC).

Video streaming and sensor data are distributed through separated VLAN in order to handle this data independently, isolating multicast and broadcast traffic.

The network has been developed in order to be fault tolerant, so if there is any link failure, the video streams are redirected through any other link. In order for this system to work, load balancing and extra bandwidth in the links has been considered.

\section{DATA CAPTURE}

Commercial video servers have evolved towards DSP (Digital Signal Processor) architectures. DSP design is costly in hardware and software. In a DSP architecture, video compression and encapsulation must be implemented with DSP oriented software. This software is often the most expensive and closed part of the system. Currently, some DSP systems are controlled by a simple operating system. This OS allows reusing code between

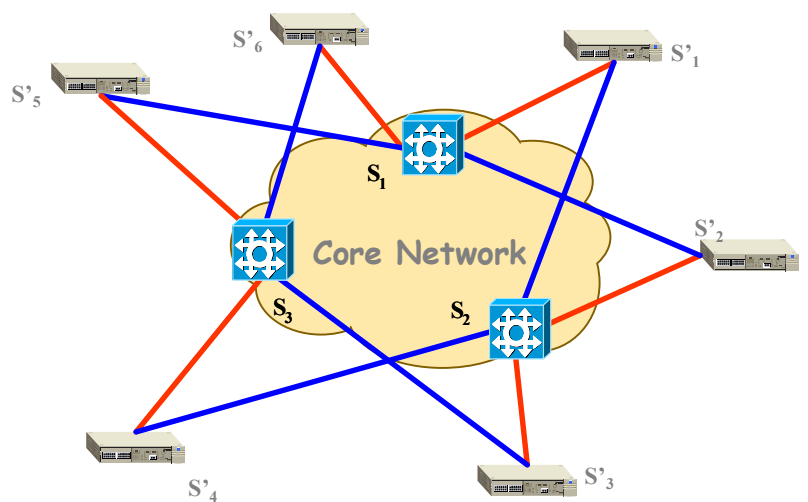

different projects. However, system calls, compilers and libraries are not standard and it is difficult to port an existent multimedia application from another operating system to the DSP system. The video servers that have been developed within the project are based on a x86 architecture.

The main tasks carried out by the video servers are the following: (i) Video capture from analogical sources, as are the cameras. The developed video servers include standard video acquisition cards, that provide raw signals to be encoded; (ii) Video compression, using different standards, here we have used VLC and MPEG4 due to the quality and bandwidth consumption. Usually urban traffic video management systems when working with digital video information use low quality compression schemas like MJPEG or proprietary techniques, or high quality but with high bandwidth consumption, such as MPEG2; (iii) Packetize the compressed video and attach timestamps within the packets, using RTP/RTCP and (iv) Transmit packets over the communication network, to the multicast address fixed by the Stream Management Server.

The video server developed in this project also includes the acquisition of data from different sensors and traffic lights that are sent back to the TMC. Additionally there is a mobile camera attached to each server and the video server relays remote PTZ (Pan-Tilt-Zoom) control information from the console to the camera motor by means of a serial interface (RS-232/422/485).

\section{VIDEO REPRODUCTION}

The visualization process can be performed in real-time or on-demand accessing the storage server. A typical group of cameras is composed by five cameras (one mobile) in one pole attached to a video server.

Different terminals can be used for displaying the information, the three mainly used are: 


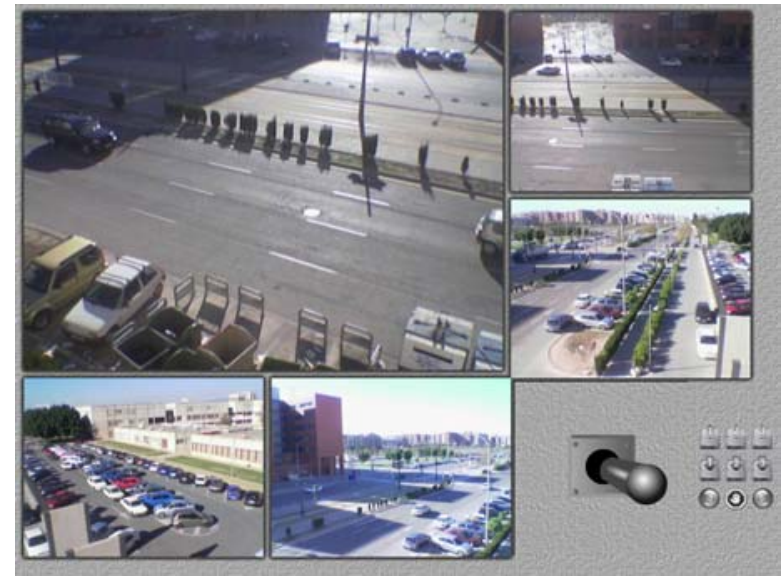

Figure 3. Management console screenshot

- The Management Console, which can receive the streaming video from a video server and reproduce the information as depicted in figure 3 . The console can access to live or stored video information.

- A Videowall in the TMC, where many cameras are presented simultaneously and sequentially, managed by the operators.

- Internet terminals, which can access through a public web page low quality video of selected cameras (not all), so the citizens can control and monitor the state of the traffic in certain points in the city.

\section{DATA DELIVERY}

Figure 4 represents the architecture of the video streaming delivery architecture, from the cameras, connected to the video servers, to the TMC. Due to the utilisation of multicast communication, the Video Stream Management Server (VSMS) allows the introduction of several applications and services.

This server controls the multicast addresses to which the video servers send the video information. Besides VSMS associates different terminals with these multicast addresses, keeping track of the used addresses.

The main components of the architecture that can receive simultaneously the information from the video servers deployed in the city streets are: (i) management console, (ii) general videowall of the TMC, (iii) Storage server, and (iv) artificial vision server. The Web server is directly fed from the storage server. There exists the possibility to add new services that can receive the video streaming information.

\section{CONCLUSSIONS}

In this paper we have described a real-time streaming solution for urban traffic monitoring. The basic system features are: (i) TCP/IP network, (ii) scalability to hold enough cameras to cover a large city, (iii) high quality

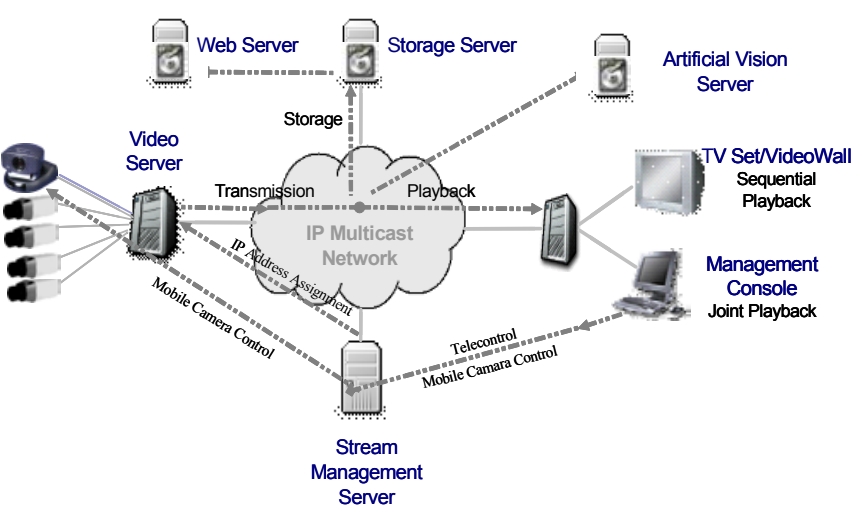

Figure 4: Video streaming delivery architecture

video compression standards (MPEG4), (iv) utilisation of standard protocols and (v) multicast delivery.

The system is fully operative and the number of installed cameras is currently being increased. The development of the system proves the viability and scalability of COTS philosophy for massive streaming video systems for urban traffic control. Compression algorithms, buffering techniques and additional protocols are the future work for this project.

\section{REFERENCES}

[1] G. Kogut, M. Trivedi, "Maintaining the Identity of Multiple Vehicles as They Travel Through a Video Network," IEEE Int'l Conf. On Intelligent Transportation Systems, Oakland, (CA), 2001

[2] T. Brett, M. Trivedi, "A Novel Interactivity Environment for Integrated Intelligent Transportation and Telematic Systems", IEEE Int'l Conf. On Intelligent Transportation Systems, 2002

[3] A. Zhang, Y. Song and M. Mielke, "NetMedia: Streaming Multimedia Presentations in Distributed Environments", IEEE Multimedia, pp.: 56-73, v.9, n.1, 2002,

[4] S. Velastin, et. al, "A flexible communications protocol for a distributed surveillance system", Journal of Networks and Computer Applications, vol 27, pp 221/253, 2004.

[5] J. C. Guerri , C. Palau, A. Pajares and M. Esteve, "A realtime e-learning system via satellite based on JMF and Windows Media, Proceedings of the tenth ACM International Conference on Multimedia, Dec. 2002.

[6] H. Radha, Y. Chen, K. Parthasarathy and R. Cohen, "Scalable Internet vides using MPEG4", Signal Processing: Image Communication, vol 15, pp: 95-126, 1999.

[7] The Videolan project, http://www.videolan.org 